\title{
Forensic Identification of Salvia divinorum and Salvinorin A
}

\author{
Dana Nontell and Douglas Armstrong \\ Department of Physical Sciences \\ Olivet Nazarene University \\ Bourbonnais, IL 60914
}

Received: May 13, $2010 \quad$ Accepted: September 10, 2010

\begin{abstract}
Salvia divinorum is a member of the mint family that is growing in popularity in the United States and many other countries as a hallucinogenic drug. Because of this, numerous studies have been conducted in identifying the plant material. Some of the most common methods include UV/Vis, TLC, and GC/MS. This article discusses these methods, as well as two other common identification methods proven to be ineffective towards salvia. An extraction method is also discussed.
\end{abstract}

\section{INTRODUCTION}

The plant Salvia divinorum, more commonly known as salvia, has recently gained popularity in the United States. It is a perennial herb in the mint family found in certain areas of Oaxaca, Mexico [1]. Its popularity is due to its hallucinogenic effects, which are attributed to the chemical Salvinorin A found in the plant. The chemical structure of salvinorin $A$ can be seen in Figure 1.

Salvia is often compared to cannabis due to its similar effects, which include perceptions of bright light, vivid colors and shapes, body and object distortions, dysphoria, uncontrolled laughter, a sense of loss of body, overlapping realities, hallucinations, incoordination, dizziness, and slurred speech [1].

Currently, salvia is not controlled under the Federal Controlled Substances Act; however, in the United States, there have been thirteen different states that have<smiles>COC(=O)[C@H]1CC(OC(C)=O)C(=O)[C@@H]2[C@@H]3C[C@H](c4ccoc4)OC(=O)[C@H]3CC[C@]12C</smiles>

Figure 1. Chemical structure of Salvinorin A.

enacted legislation placing regulatory controls on Salvia divinorum and/or salvinorin A [1]. Nine of those states have placed the plant into schedule I of state law, and the other four states have made forms of legislation restricting the distribution of the plant. 


\section{PHARMACOLOGY}

Salvia is typically either chewed or smoked. When chewed, the leaf mass and juice are maintained within the cheek area with absorption occurring across the lining of the oral mucosa. The effects appear within five to ten minutes [1]. When smoked, dried leaves and/or extract-enhanced leaves alleged to be enriched with salvinorin $A$ are used. When smoking pure salvinorin $A$, at a dose of 200-500 micrograms, the effects can be seen within 30 seconds and lasts about a half hour [1]. There is no known medical use for salvia at this time.

Salvinorin $A$ is a neoclerodane diterpene found primarily in the leaves, and to a lesser extent in the stems [1]. Many other substances have been extracted from salvia, including salvinorin $\mathrm{B}$ and $\mathrm{C}$; however, they have not been proven to produce hallucinogenic effects. Other scientific studies show that salvinorin $A$ is a potent and selective kappa opioid receptor agonist, similar to certain other hallucinogenic drugs; however, salvinorin $A$ does not activate the serotonin $2 \mathrm{~A}$ receptor, which mediates the effects of other schedule I hallucinogens [1].

\section{PRELIMINARY TESTS}

Salvia is often compared to cannabis; however, considering microscopy, there are many differences, which can be seen in Table 1.
In forensics, cannabis can be easily identified from its leaves. The upper part of the leaf is dark green in color and contains sparse hairs; the lower portion of the leaf is paler in color and is more densely hairy [2]. No other plant has leaves similar to cannabis. Salvia is a very common-looking plant. It has no outstanding characteristics that separate its looks from other plants. This is why microscopy is not used to identify salvia.

Color tests also show differences between salvia and cannabis. Many forensic scientists use the Duquenois-Levine test to test for cannabis. The DuquenoisLevine test can be made by adding $2.5 \mathrm{ml}$ of acetaldehyde and $2.0 \mathrm{~g}$ of vanillin to $100 \mathrm{ml}$ of $95 \%$ ethanol. This test then requires $1 \mathrm{ml}$ of this solution to be mixed with cannabis and shaken for one minute. One milliliter of concentrated hydrochloric acid is added and the solution is agitated gently. Three milliliters of chloroform are added, and the color produced for a positive I.D. of cannabis is a deep purple [3]. This test, like many other color tests, does not give a unique color when performed on salvia. There is currently no known color test that can be used to identify salvia.

The only preliminary test that does shed some light on whether or not a substance contains salvinorin $A$ is UV/Vis. This test is a fast and easy way to initially screen for salvinorin $A$, which will absorb light at 210nm [4].

\begin{tabular}{|l|l|l|}
\hline Description & Salvia & Cannabis \\
\hline Height & Over 1 meter & Between 2 to 7 meters \\
\hline Stems & Hollow, square & Woody, rough, round \\
\hline Leaves & Large, green & $\begin{array}{l}\text { Dark green (top), pale green (bottom), hairy, 5 to } 9 \\
\text { finger-like leaflets }\end{array}$ \\
\hline Flowers & White, purple calyces & Green, small (male), leafy spikes (female) \\
\hline
\end{tabular}

Table 1. Comparison of Salvia and Cannabis. [1, 2] 


\section{EXTRACTION}

Salvia is the only known natural source of salvinorin A [4]. Because of this and because salvia cannot be identified by microscopic methods, salvia is identified by the presence of salvinorin A. Often times, salvia is found as fresh and dried leaves. In order to test for salvinorin $A$, an extraction must be done. A simple extraction with a common organic solvent yields a solution that is rich in salvinorin A [4]. Because of the plant's dark green color, pigments are also extracted. This problem can be fixed by adding activated charcoal for a short period of time [4]. This solution can them be evaporated down and then purified by recrystallization methods to reveal purified salvinorin $A$ [4]. This purified salvinorin $A$ is the substance that can be analyzed by other instrumental methods.

\section{THIN LAYER CHROMATOGRAPHY}

Thin layer chromatography (TLC) is also an important test used in forensics. It can be used to separate solutions or to confirm an unknown with a known by looking at the $R_{f}$ values. When working with actual plant material, one study has shown that salvinorins can be found in the trichomes of the abaxial, young leaves; however, the roots, internal stem tissue, cotyledons, and corolla do not contain salvinorins [5]. This study was done on a silica gel plate, which was developed in ethyl acetate/hexanes (1:1). After the solution ran up the plate, the terpenes were visualized by spraying the plates with a vanillin reagent $(50 \mathrm{ml}$ ethanol, $0.3 \mathrm{ml}$ sulfuric acid, and $1 \mathrm{~g}$ vanillin). This was then heated at $110^{\circ} \mathrm{C}$, where pinkishpurple spots were formed on the plate [5]. This method can also be used with tetrahydrocannabinol (THC) and cannabinol, both of which have different $R_{f}$ values than salvinorin $A$. This is important, because TLC can be used to identify whether or not

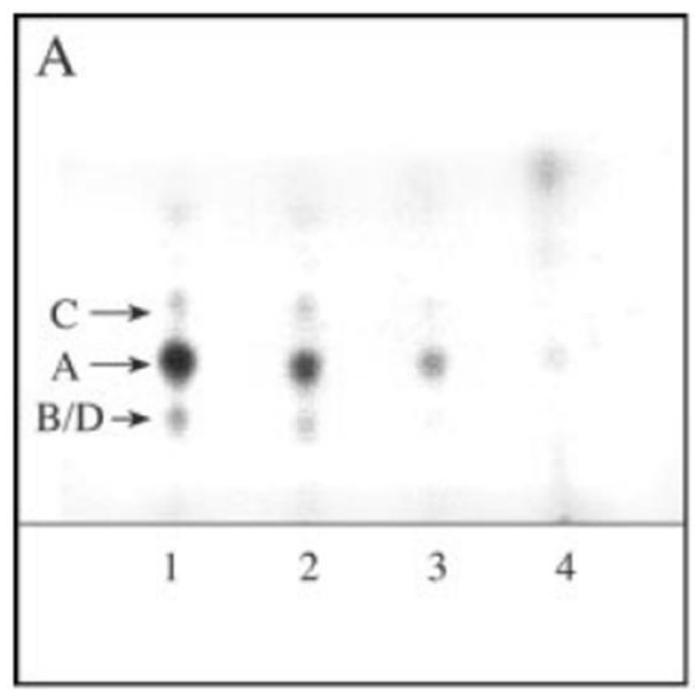

Figure 2. Thin layer chromatogram of sequential chloroform extracts of fresh leaves, where $A=$ salvinorin $A, B / D=$ salvinorin $B$ and salvinorin $D$, and $C=$ salvinorin C. Lane 1, first 30-s extract; lane 2 , second 30-s extract; lane 3, third 30-s extract; lane 4, 2-h chloroform extract prepared from previously extracted leaves after they had been dried and powdered. [5].

an unknown plant material is actually cannabis or salvia. An example of the use of TLC can be seen in Figure 2.

\section{GAS CHROMATOGRAPHY- MASS SPRECTROMETRY}

Gas chromatography-mass spectrometry, or GC-MS, is a very useful tool in drug identification. GC-MS is one of the most common instruments used in forensic drug detection due to its ease of use and reproducibility. The GC part separates the sample into its individual compounds, and the MS part analyzes each compound. The mass spectrum is obtained by ionizing each compound to create fragments, which are then measured by their mass-to-charge ratios. The mass spectrum of a compound is unique to that particular compound. Forensic scientists identify unknown samples by comparing the mass spectrum 


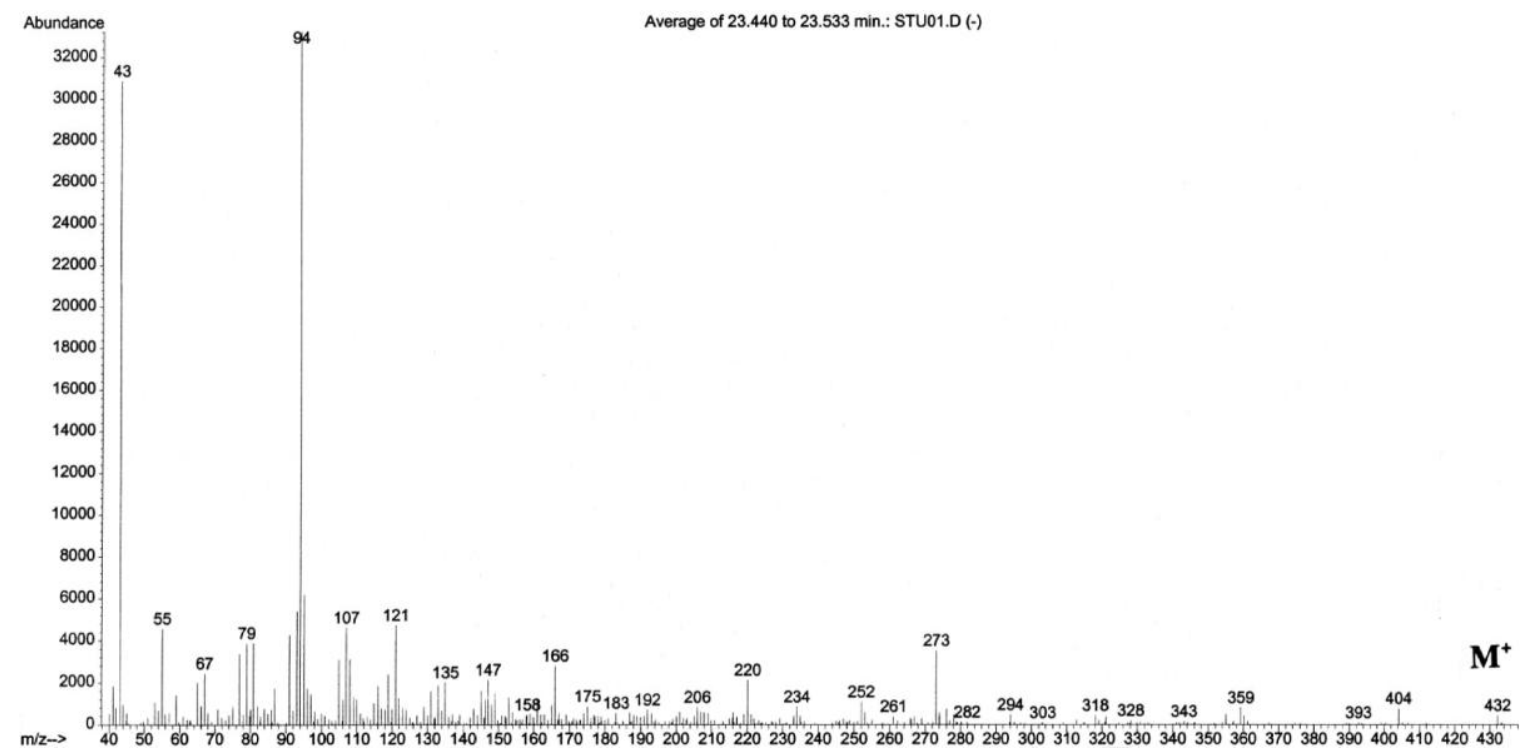

Figure 3. The 70-eV Mass Spectrum of Salvinorin A. [7].

of the unknown sample to a known drug's mass spectrum. The mass spectrum of salvinorin A can be seen in Figure 3.

When using GC-MS in identifying salvia, different organic solvents can be used, the most common being methanol and chloroform. Often times, the active constituent, salvinorin $A$, is not the only peak that shows up. Other peaks may include salvinorin $B$, salvinorin $C$, and different pigments, depending on how long the plant material remains in the solvent. An important note about using GC alone is that some compounds may have similar retention times as other compounds. This is why many scientists often couple GC with MS; MS is a confirmatory test.

Because salvinorin $A$ must be extracted from salvia, GC-MS has a low sensitivity when detecting salvinorin $A$. This leads forensic scientists to create methods that utilize a splitless injector, in hopes of making the peaks from the sample stronger. One such method was done on an Agilent Technologies $6890 \mathrm{~N}$ instrument, equipped with an autosampler and 5793 mass spectrometric detector. This method was run on a Restek $5 \mathrm{Ms}$ column $(30 \mathrm{~m} \mathrm{x}$ $0.25 \mathrm{~mm})$. The injector volume was a splitless $1 \mu \mathrm{L}$ and the injector temperature was $250^{\circ} \mathrm{C}$. The temperature gradient was $40-300^{\circ} \mathrm{C}$ over $15 \mathrm{~min}$ then held at $300^{\circ} \mathrm{C}$ for 12 min [6]. This method was in fact able to identify salvinorin $A$; however it was very weak, due to the reasons mentioned above.

\section{CONCLUSION}

The use of Salvia divinorum is on the rise, due to its legal status in many states, ease of use, and hallucinogenic properties. Salvia is most popular with young adults, years 18 to 25 , with more common use in males than females [1]. Salvia has no approved medical use in the US, and therefore, is currently being closely monitored with a possibility to place it in schedule 1 by the Drug Enforcement Administration. Known uses of detection include UV/Vis, TLC, and GC/MS. More needs to be known about Salvia divinorum, and more studies should be conducted both in the medical field and in forensic detection.

\section{REFERENCES}

1. Drug Enforcement Administration. (November 2008). Salvia divinorum 
and salvinorin A. Washington, D.C.: Office of Diversion Control, Drug \& Chemical Evaluation Section.

2. Keith Turnbull Research Institute. (January 1998). Marijuana: State prohibited weed. Department of Primary Industries.

3. National Institute of Justice. (n.d.). Color test reagents/Kits for preliminary identification of drugs of abuse. Law Enforcement and Corrections Standards and Testing Program.

4. Cawrse, B.M., Hall, A.B. (2008). Forensic chemical analysis and characterization of Salvia divinorum and salvinorin $A$. White Plains, NY: Boston University School of Medicine.

5. Siebert, D.J. (2 February 2004). Localization of salvinorin $A$ and related compounds in glandular trichomes of the psychoactive sage, Salvia divinorum. Annals of Botany, 93, 763-771.

6. Medana, C., Massolino, C., Pazzi, M., \& Baiocchi, C. (9 November 2005). Determination of salvinorins and divinatorins in Salvia divinorum leaves by liquid chromatography/multistage mass spectrometry. Rapid Communications in Mass Spectrometry, 20, 131-136.

7. Giroud, C., Felber, F., Augsburger, M., Horisberger, B., Rivier, L., \& Mangin, P. (24 March 2000). Salvia divinorum: $A$ hallucinogenic mint which might become a new recreational drug in Switzerland. Forensic Science International, 112, 143-150.

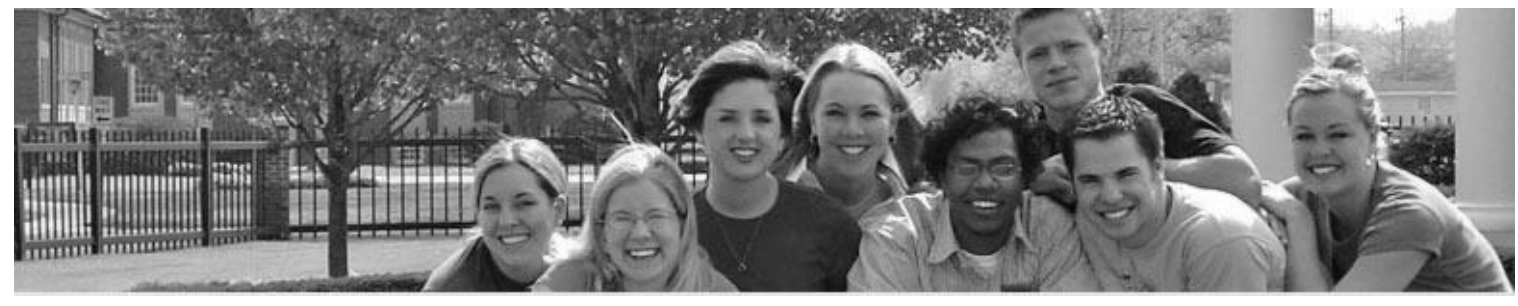

\section{Olivet Nazerene University Bourbonnais, Illinois-just 50 minutes south of Chicago's Loop}

Education with a Christian Purpose. Since 1907, Olivet Nazarene University has made this more than a motto, but a mission. At Olivet, considered one of the nation's premier Christian colleges, faith is at the heart of superior academics, athletics, social atmosphere and ministry opportunities.

Here, students not only learn how to make a living; they learn how to live. Since Olivet's founding, more than 20,000 degrees have been granted to graduating students. Whether their chosen fields are in medicine, business, education, ministry or a myriad of other professions, Olivetians make a difference in the world for Christ and His kingdom.

At Olivet, ambitious dreams meet uncommon opportunity.

http://www.olivet.edu/ 


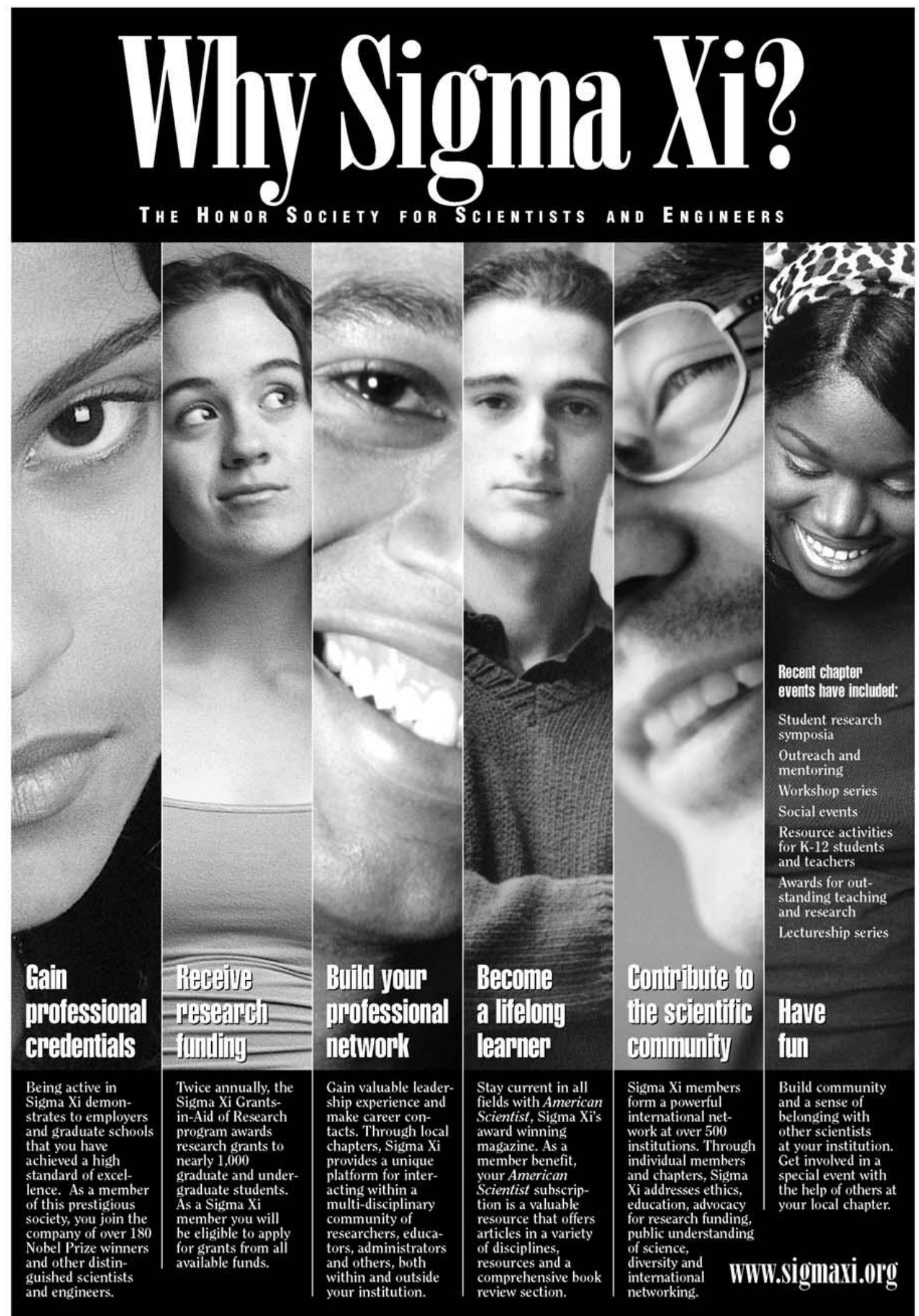

Sigma Xi, The Scientific Research Society • 98 Alexander Drive • P.O. Box 13975 • Research Triangle Park, NC 27708 • 818-549-4691 • 800-243-6534 